\title{
« Eure Majestät fragen mich etwas spät » (à propos de Charles I ${ }^{\mathrm{er}}$ )
}

" Euere Majestät fragen mich etwas spät » (ad Karl I.)

« Euere Majestät fragen mich etwas spät » (about Charles I)

\section{Christopher Brennan}

\section{OpenEdition}

\section{Journals}

Édition électronique

URL : http://journals.openedition.org/austriaca/351

DOI : 10.4000/austriaca.351

ISSN : 2729-0603

Éditeur

Presses universitaires de Rouen et du Havre

\section{Édition imprimée}

Date de publication : 1 décembre 2018

Pagination : $77-102$

ISBN : 979-10-240-1354-1

ISSN : 0396-4590

\section{Référence électronique}

Christopher Brennan, « « Eure Majestät fragen mich etwas spät » (à propos de Charles |er) », Austriaca [En ligne], 87 | 2018, mis en ligne le 01 mars 2020, consulté le 28 janvier 2021. URL : http:// journals.openedition.org/austriaca/351 ; DOI : https://doi.org/10.4000/austriaca.351 
Christopher BRENNAN

Londres

\section{« Eure Majestät fragen mich etwas spät » (à propos de Charles ${ }^{\text {er }}$ )}

Avant même le début de la première guerre mondiale, il n'était pas rare d'entendre ou de lire que l'Empire austro-hongrois périrait avec François-Joseph ${ }^{1}$. Ce dernier avait déjà 83 ans au début des hostilités. Son successeur, après l'assassinat de François-Ferdinand, est son petitneveu, l'archiduc Charles-François-Joseph - un jeune homme de 26 ans sans envergure apparente, peu connu du public dans son propre pays et presque entièrement inconnu à l'étranger. Alors que l'accession au trône du futur empereur Charles I ${ }^{\text {er }}$ (Charles IV pour les Hongrois) est sans doute proche et que l'Empire habsbourgeois s'embourbe dans une guerre désastreuse, l'héritier ne bénéficie d'aucune préparation sérieuse pour son rôle à venir ; il ne la demande pas non plus. Il se distingue sur le champ de bataille, prenant parfois des risques insensés, mais n'acquiert aucune connaissance diplomatique, administrative ou politique $^{2}$. Ainsi, lorsque la fin de la guerre approche, après presque deux ans de règne, Charles avait déjà failli et il continua à faillir dans presque toutes ses entreprises civiles, particulièrement en ce qui concerne la réforme de l'Empire si nécessaire et tant attendue. En contrepoint d'une situation militaire devenue satisfaisante - en effet, l'AutricheHongrie a atteint tous ses objectifs de guerre à la fin de 1917 -, le chaos règne à l'intérieur des terres, particulièrement du côté autrichien : répression, censure, réfugiés, famine, grèves, tensions nationales, lassitude et désespoir. Mais, malgré les craintes de la famille impériale et royale, du gouvernement, de l'aristocratie et de la bourgeoisie, la révolution n'éclate pas. Les sociaux-démocrates autrichiens se sont d'ailleurs toujours montrés coopératifs. Et la monarchie perdure, tant

1. Christopher Brennan, Reforming Austria-Hungary: Beyond his control or beyond his capacity? The domestic policies of Emperor Karl I: November 1916 - May 1917, thèse de doctorat, London School of Economics, Faculté d'histoire internationale, novembre 2012, p. 105.

2. Ibid., p. 16-49. 
bien que mal. Reste que le sort de l'Autriche-Hongrie est lié à celui de l'Allemagne, surtout après la découverte par Berlin des négociations secrètes avec les Alliés menées par Sixte de Bourbon-Parme, le beaufrère de Charles, à l'instigation de ce dernier. Il est d'ailleurs révélateur qu'aucun des pourparlers initiés ou soutenus par Charles pendant son règne n'envisage une paix séparée, c'est-à-dire sans les Allemands, avec les Alliés. Charles n'est ni pangermaniste ni prussophile, mais les Habsbourg vaincront (à la Pyrrhus) ou couleront avec les Hohenzollern. Chaque velléité d'échapper à cette alliance fatale, cette Nibelungentreue, est étouffée par l'illusion d'une victoire potentielle des empires centraux : Caporetto fin 1917 et, en 1918, les traités de BrestLitovsk et de Bucarest, la préparation d' une offensive finale contre une Italie au bord du gouffre, l'offensive du printemps des Allemands. Ces motifs d'optimisme détournent aussi Charles de la refonte de son pays chancelant $^{3}$. Il est évident que la réorganisation de la double monarchie dépendra de l'aboutissement de la guerre. Mais dès la fin de l'été 1918, l'impasse est totale. La courte agonie de l'Autriche-Hongrie et des Habsbourg commence.

Après le 8 août 1918, la fameuse "journée noire » de l'armée allemande, et surtout la demande de cessez-le-feu de la Bulgarie (qui parvient à Charles le matin du 25 septembre), suivie de l'acceptation de l'armistice bulgare par les Alliées le 29, le sort des empires centraux est scellét. Les Autrichiens sont mal en point, même si l'armée continue de se battre valeureusement et loyalement dans des conditions épouvantables : en septembre, on estime entre 230000 et 250000 le nombre total de déserteurs, de prisonniers de guerre évadés et de permissionnaires restés à l'écart de leurs unités ${ }^{5}$. Pourtant, Charles semble conserver un certain flegme. Le comte Arthur Polzer-Hoditz, l'ami d'enfance et ancien directeur de cabinet de l'empereur, en

3. Pour un résumé de la « carrière » de Charles entre 1914 et 1917 en tant qu’héritier sans direction, puis brièvement, empereur avec une certaine marge de manœuvre, voir : Christopher Brennan, "'Hesitant Heir and Reluctant Ruler': Karl I/IV of Austria-Hungary During the Great War ", dans Matthew Glencross et Judith Rowbotham (dir.), Monarchies and the Great War, Basingstoke, Palgrave Macmillan, 2018, p. 87-118.

4. Voir Arthur Freiherr Arz von Straußenburg, Zur Geschichte des Großen Krieges 1914-1918. Aufzeichnungen, Wien, Rikola, 1924, p. 302-303 ; Neue Freie Presse, Morgenblatt (désormais NFPM), Vienne, 28 septembre 1918, p. 1.

5. Voir Jean Sévillia, Le dernier empereur. Charles d'Autriche 1887-1922, Paris, Perrin, 2009, p. 186 ; Bernard Michel, La chute de l'Empire austro-hongrois 1916-1918, Paris, Robert Laffont, p. 205. 
témoigne, alors que les nouvelles de Sofia viennent d'atteindre Baden, le quartier général de l'armée impériale et royale, ramené près de Vienne par Charles :

L'empereur était très grave, mais contrairement à l'humeur de tous ceux qui circulaient en hâte et attendaient dans la maison, il ne manifestait pas le moindre signe de nervosité. Il était calme; il parlait avec clarté et détermination. Ce qu'il avait toujours redouté, mais ce à quoi personne n'avait voulu croire commençait à poindre à l'horizon : l'issue de la guerre serait défavorable aux puissances centrales par suite de l'intervention américaine ${ }^{6}$.

L'empereur a beau rester calme en surface, le ministre Alexander von Spitzmüller se souvient que Charles était déjà très déprimé pendant tout le mois de septembre ${ }^{7}$. Le 27 septembre, un Conseil de la couronne a lieu dans la capitale autrichienne. Le ministre commun des Affaires étrangères, le comte István Burián, annonce que « la catastrophe bulgare a fait considérablement empirer notre situation à tout point de vue, en particulier militaire et politique ». Mais il déclare, inflexible : "Le principe suprême doit être le suivant : maintien de la forme dualiste de la Monarchie. » Autre dinosaure de la politique obstinée de la Hongrie, l'indéfectible vétéran Sándor Wekerle, le Premier ministre venu de Budapest, approuve son collègue. En résumant la séance, Charles insiste sur la nécessité d'un nouvel effort pour inciter Berlin à accepter la paix et d'une réforme constitutionnelle immédiate 8 . Aucune objection. Mais ce que Charles entend par cette " reconstruction intérieure de l'Autriche " n'est pas clair, pas plus dans sa tête que dans celle de ses ministres : s'agit-il de l'Empire dans son entier ou simplement

6. «Der Kaiser war sehr ernst doch war bei ihm zum Unterschied von der Stimmung aller der im Hause herumhastenden und wartenden Menschen keine Spur von Nervosität zu bemerken. Er war ruhig, sprach klar und bestimmt. Was er immer befürchtet hatte und was man ihm niemals habe glauben wollen, beginne nun einzutreten: Die Entscheidung zuungunsten der Mittelmächte als Folge des Einschreitens Amerikas» (Arthur Graf Polzer-Hoditz, Kaiser Karl. Aus der Geheimmappe seines Kabinettschefs, Zürich/Leipzig, Amalthea, 1929, p. 553-554).

7. Memoirs of Alexander Spitzmüller Freiherr von Harmersbach (1862-1953)..., Carvel de Bussy (trad. et éd.), Boulder, East European Monographs, 1987, p. 189.

8. Miklós Komjáthy, Protokolle des Gemeinsamen Ministerrates der ÖsterreichischUngarischen Monarchie (1914-1918), Budapest, Akadémiai Kiadó, 1966, doct. 39, p. 681683, 685, 687 : «Die bulgarische Katastrophe hat unsere Lage in jeder Hinsicht namentlich in militärischer und politischer ganz bedeutend verschlimmert. [...] Oberstes Grundprinzip muss lauten: Beibehaltung der dualistischen Gestaltung der Monarchie. » 
de la partie autrichienne, la Cisleithanie ? Et d'ailleurs, comment s'y prendre ? Ce sujet a beau bouillonner depuis la révolution de 1848 (au moins) et surtout depuis la conclusion peu satisfaisante du Compromis de 1867, Charles n'est pas un grand théoricien. Son premier message en tant qu'empereur-roi en novembre 1916, son couronnement à Budapest le mois suivant, ses choix de ministères, ses rares incursions dans la politique intérieure du pays ainsi que son allocution devant le parlement de Vienne le 31 mai 1917, ont largement déçu. Ce qu'il dit et pense en privé ne perce presque jamais dans le public ${ }^{9}$. Tant que l'échéance peut être repoussée, Charles le fait allègrement. Mais, après plus d'une année stérile sous l'administration principalement apolitique du bureaucrate Ernst Seidler von Feuchtenegg entre juin 1917 et juillet 1918, l'empereur a senti que le vent tournait et a nommé Max von Hussarek von Heinlein en tant que Premier ministre le 26 juillet. Peu après, il choisit comme ministre commun des Finances, Alexander von Spitzmüller, un homme sensé qui souhaite la création d'un État trialiste avec les portions sud-slaves de l'Autriche, de la Hongrie, en plus de la Bosnie-Herzégovine ${ }^{10}$. Pour Charles, ceci représentait un pas vers la fédéralisation de l'Autriche, même si Hussarek n'a pas la réputation d'un réformateur ; le rigide Seidler devient en outre le chef de cabinet de l'empereur.

Le 14 septembre, Burián a envoyé une note à tous les belligérants afin "d'entamer une conversation [...] sur les principes fondamentaux d'une paix à conclure ». Il n'a pas manqué d'y critiquer les velléités (tardives) de l'Entente qui viseraient au « démembrement de l'AutricheHongrie ", mais a rappelé aussi que le Premier ministre britannique David Lloyd George et le président américain Woodrow Wilson ont par la suite tenu des discours avec lesquels son pays s' accorderait ${ }^{11}$. L'espoir est donc là. Les Autrichiens pensent qu'en changeant de constitution et en se conformant aux quatorze points de Wilson du 8 janvier 1918, ils pourront se sauver ${ }^{12}$. Le dixième point, en particulier, stipulait : « Aux

9. L'impératrice Zita (voir Gordon Brook-Shepherd, The Last Habsburg, London, Weidenfeld \& Nicolson, 1968) et le comte Polzer-Hoditz en particulier ont rapporté leurs souvenirs d'un empereur déterminé à reformer et fédéraliser l'Empire.

10. Bernard Michel, op. cit., p. 203-204 ; Memoirs of Spitzmüller, op. cit., p. 192 et 196-197.

11. Reproduction du texte original en français dans la NFPM, 15 septembre 1918, p. 2.

12. Baron Stefan Kray, Im Dienste der Kabinettskanzlei während des Weltkrieges, Episoden und Charakterbilder aus dem Leben der Kaiser Franz Josef und Karl. Reflexionen eines ehemaligen Hofsekretärs der k.u.k. Kabinettskanzlei, Budapest, Révai, 1937, p. 170. Les 14 points furent publiés dans la presse viennoise le 10 janvier 1918 ; voir lédition de 
peuples d'Autriche-Hongrie, dont nous désirons voir sauvegardée et assurée la place parmi les nations, devra être accordée au plus tôt la possibilité d'un développement autonome. » Les Autrichiens ignorent à ce moment que les Américains ont d'ores et déjà renoncé à traiter avec eux, ce qui explique leur naïveté et leur aveuglement. Lorsque Robert Lansing, le secrétaire d'État américain, reçoit la note de Burián des mains de la légation suédoise à Washington, il confie (en français) que " la réponse ne sera pas très encourageante ${ }^{13}$ ". Effectivement, les nationalités, soutenues par les Alliés, sont à deux doigts de l'indépendance et l'Allemagne au bord de la ruine. L'historien français Jean-Paul Bled commente cette démarche : «Un an, voire seulement quelques mois plus tôt, les Alliés de l'Entente auraient accueilli avec intérêt une demande austro-hongroise de paix séparée. Ils y auraient vu un moyen d'affaiblir l'Allemagne. Aujourd'hui la situation a changé. Ces pays ont pris des engagements envers les comités nationaux ${ }^{14}$. " Lansing, logiquement, recommande au président Wilson de repousser les propositions autrichiennes (mais non les allemandes) afin d'en finir avec les Habsbourg. Par conséquent, Vienne - contrairement à Berlin, dont l'ambassade à Vienne aurait fatalement saboté l'entreprise autrichienne en diffamant Charles $^{15}$ - ne reçoit aucune réponse de Washington. Pourtant, l'Autriche-Hongrie, l'Allemagne et l'Empire ottoman ont officiellement annoncé le 5 octobre leur décision d'approcher les Américains en vue d'un armistice général et de négociations pour la paix ${ }^{16}$.

Mais Charles continue de tenter cette réorganisation, sans que quiconque sache véritablement comment s'y prendre. Un Conseil des ministres secret le 2 octobre ne fait qu'entériner les divergences entre les membres du gouvernement. Hussarek espère encore : «En ce qui concerne maintenant les quatorze points de Wilson, à bien des égards ils ne nous sont pas défavorables ${ }^{17}$. " Spitzmüller va plus

ce jour de la NFPM, p. 2-3 : «Den Völkern Österreich-Ungarns, deren Platz unter den Nationen wir geschützt und gesichert zu sehen wünschen, soll die erste Gelegenheit zu autonomer Entwicklung gewährt werden. »

13. Stephan Burián, Drei Jahre: aus der Zeit meiner Amtsführung im Kriege, Berlin, Ullstein, 1923, p. 295.

14. Jean-Paul Bled, Lagonie d’une monarchie. Autriche-Hongrie, 1914-1920, Paris, Tallandier, p. 400 .

15. Bernard Michel, op. cit., p. 204.

16. Arthur Freiherr Arz von Straußenburg, op. cit., p. 308-310; Neue Freie Presse, Abendblatt (NFPA), 5 octobre 1918, p. 1 ; NFPM, 10 octobre, p. $1 ; 13$ octobre, p. 1.

17. Miklós Komjáthy, Protokolle, op. cit., doct. 40, p. 690 : «Was nun die vierzehn Wilsonschen 
loin et déclare que seule une troisième entité sud-slave peut satisfaire Slovènes, Croates et Serbes. Burián et Wekerle sont indignés par ces $\operatorname{propos}^{18}$. Les désaccords entre Hongrois et Autrichiens sont insurmontables. Les balbutiements de Charles sont édifiants : le jour du Conseil de la couronne du 27 septembre, il avait convoqué le baron Johann von Eichhoff, ancien conseiller constitutionnel de FrançoisFerdinand $^{19}$. Comme s'il s'agissait d'une banale affaire administrative, Charles lui avait demandé avec sa légèreté coutumière : "À l'époque, vous aviez travaillé avec l'Archiduc François ; comment envisageait-il la nouvelle constitution ? » Demeuré coi, Eichhoff n’osa prononcer les mots qu'il avait sur le bout de sa langue : "Votre Majesté me le demande un peu tard ${ }^{20}$.»

Hussarek ne s'y prend guère mieux. Lorsqu'il confie secrètement à Vlastimil Tusar (de facto le représentant du Conseil national tchèque à Vienne) que la paix est proche, Tusar demande à connaître la nouvelle position du gouvernement sur la réforme intérieure du pays. Il n'y en a pas. Hussarek répète sa dernière déclaration au Parlement selon laquelle une autonomie limitée sera octroyée aux nationalités autrichiennes sous le sceptre des Habsbourg ${ }^{21}$. Quel intérêt pour les Tchèques bientôt libres de l'écouter encore ? Même scénario au Parlement : les députés des nationalités annoncent leur sécession imminente, mais le gouvernement ne leur offre que des miettes en retour ${ }^{22}$. Les discussions à huis clos entre le gouvernement et les groupements nationaux n'apportent rien non plus ${ }^{23}$.

De son côté, Charles reçoit les chefs de tous les partis et de toutes les nationalités des deux chambres des députés à Baden le samedi 12 octobre, afin de discuter de la reconstruction fédérale de l'Autriche, la question la plus importante depuis la guerre de Succession de 1740 d'après la Neue Freie Presse. Les discussions, qui durent cinq heures, sont ouvertes,

Punkte anbelange, so seien dieselben in mancher Hinsicht für uns nicht ungünstig. »

18. Ibid., p. 693-695; Memoirs of Spitzmüller, op. cit., p. 194-197.

19. Wien, Staatsarchiv, Kriegsarchiv, Nachlass Johann von Eichhoff, B/874, 150, Mémoires, Von Miramar nach St. Germain, p. 36, p. 42-43. Les mémoires d'Eichhoff sont partiellement publiés dans : Peter Broucek (éd.), Theodor Ritter von Zeynek: Ein Offizier im Generalstabskorps erinnert sich, Wien/Köln/Weimar, Böhlau, 2009, annexe 2, p. 317-328.

20. Johann von Eichhoff, Mémoires, op. cit., p. 42-43 : «"...] Sie haben ja damals die Sachen für Erzherzog Franz gearbeitet; wie hat er sich denn die neue Verfassung vorgestellt...?" Mir lag der schmerzliche Ausruf auf den Lippen: "Euere Majestät fragen mich etwas spät". "

21. Bernard Michel, op. cit., p. 216.

22. NFPM, 5 octobre 1918, p. 5-7.

23. Ibid., 18 octobre 1918, p. 1. 
honnêtes et informelles. Charles écoute attentivement, s'informe et veut comprendre. Les députés n'ont que le mot d'autodétermination à la bouche. Ceci influencera certes le dernier coup de dés de l'empereur, mais il s'agit encore d'une journée perdue. En effet, l'idée d'un gouvernement des peuples (qui avait bercé d'illusions les gouvernants) doit être abandonnée à cause de l'intransigeance des Slaves ; les Tchèques veulent leur propre État et n'accepteront rien de moins; les Slaves du Sud suivent le même cap ; les Polonais ne discuteront de leur avenir qu' avec les trois parties constituantes de la Pologne historique ; les Ukrainiens veulent un État autonome et réclament des morceaux de Galicie, de Bucovine et de Hongrie ; logiquement, les germanophones refusent de rester seuls à bord. Beaucoup de députés préfèrent attendre la conférence de paix ; les Slaves en particulier comptent sur l'Entente pour exaucer l'intégralité de leurs vœux ${ }^{24}$. Charles, lui, n'attendra pas. Le 15, très agité, il convoque un Conseil des ministres et décrit «le terrible chaos politique » qu'il a constaté. Ne voulant pas abdiquer, il insiste sur la nécessité d'entamer la future conférence de paix en tant qu'État constitutionnellement fédéral. Spitzmüller, qui a connaissance du travail d'Eichhoff, insiste sur la nécessité de reformer les deux moitiés de la monarchie. Il lui paraît évident que Hussarek (absent des consultations du 12) et Wekerle (absent du conseil, mais à Vienne), les deux Premiers ministres, vont démissionner. Charles, devenu passif et mal à l'aise, clôt subitement la réunion, sans annoncer de décision. Les mémoires de Spitzmüller, pourtant respectueux envers Charles, expliquent sans ambages le revirement de l'empereur : d'une part, il a peur de la réaction d'un Wekerle « insurmontable » et d'autre part, le cardinal de Vienne, Gustav Piffl, l'a supplié de garder le très catholique Hussarek ${ }^{25}$. La réforme déjà trop tardive est viciée, donc obsolète.

La fin était certes inéluctable, mais d'aucuns considèrent le 16 octobre comme le dernier clou fiché sur le cercueil de l'Empire ${ }^{26}$. En effet, ce jour vit la proclamation par Charles du manifeste impérial « $\grave{A}$ mes loyaux peuples autrichiens » pour la transformation de l'Autriche en État fédéral : «L'Autriche doit, suivant la volonté de ses peuples, devenir un État fédéral, dans lequel chaque peuple construira sa communauté

24. NFPA, 12 octobre 1918, p. 1-2; NFPM, 13 octobre, p. 1 et 3-4.

25. Memoirs of Spitzmüller, op. cit., p. 199-201.

26. Katrin Unterreiner, „Meinetwegen kann er gehen." Kaiser Karl und das Ende der Habsburgermonarchie, Wien-Graz-Klagenfurt, Molden, 2017, p. 59. 
nationale sur son territoire ${ }^{27}$. " Celui-ci, contresigné par Hussarek, qui n'a aucun pouvoir sur la Hongrie, prévoit la création de quatre États en Cisleithanie : un allemand, un tchèque, un sud-slave et un ukrainien ${ }^{28}$. Charles y voit de toute évidence la seule issue pour sauver la monarchie, alors que plus aucun peuple ne songe à une telle union sous le sceptre habsbourgeois. Preuve de son zèle réformateur et fédéraliste pour ses thuriféraires, il s'agit en fait d'un document désespéré, risible et pour finir ridiculisé. Même le fidèle Polzer, qui s'est battu en vain pour que Charles n'apparaisse pas comme signataire, écrit :

[...] nous avions eu cinquante ans pour reconstruire l'Autriche sur des bases nationales et fédérales; aujourd'hui il est trop tard. Une telle mesure, dans de telles circonstances, donne le coup de grâce à l'Autriche ${ }^{29}$.

Le manifeste n'anticipe pas la pleine réunification de la Pologne (qui comprend des territoires ukrainiens) et exclut fermement la Hongrie tout entière du projet de réforme (mais encourage involontairement et simultanément ses minorités opprimées). En effet, Charles refusa toujours d'abjurer son serment prononcé lors de son sacre à Budapest le 30 décembre 1916, selon lequel il promettait de ne jamais toucher à l'intégrité et à l'indivisibilité du royaume de Hongrie. Il semblait pourtant avoir globalement compris les enjeux de la question sudslave. Par ailleurs, la solution proposée pour le brûlant conflit germanotchèque en Bohême (un thème que Charles n'a jamais vraiment maîtrisé ou analysé) ne peut que provoquer l'ire des Tchèques, qui y voient la division du royaume de la Couronne de saint Venceslas.

Les mémoires d'Eichhoff offrent une toile de fond chaotique, voire tragicomique, à ce manifeste. Le baron fut réveillé dans la nuit du 14 au 15 octobre et appelé auprès de Charles à six heures du matin. L'empereur lui dit simplement :

27. «An Meine getreuen österreichischen Völker! [...] Österreich soll, dem Willen seiner Völker gemäß, zu einem Bundesstaate werden, in dem jeder Volksstamm auf seinem Siedlungsgebiete sein eigenes staatliches Gemeinwesen bildet. " Le texte complet du manifeste est publié à la hâte le jour suivant, dans une édition spéciale du journal officiel, Wiener Zeitung, Extra-Ausgabe, puis dans la presse le lendemain. Voir Helmut Rumpler, Das Völkermanifest Kaiser Karls vom 16. Oktober 1918. Letzter Versuch zur Rettung des Habsburgerreiches, Wien, Verlag für Geschichte und Politik, 1966.

28. NFPM, 17 octobre 1918, p. 2.

29. Arthur Graf Polzer-Hoditz, Kaiser Karl, p. 556-557. 
Refaites-moi une formulation du manifeste - Autriche allemande, Bohême, Illyrie, Galicie - et nous interviendrons contre quiconque s'y oppose. Ces troubles bolcheviques doivent cesser - mais laissez-moi la Hongrie en paix, la Hongrie est la partie endormie ${ }^{30}$.

Peu importe que Charles consulte l'expert Eichhoff à l'aube, en présence de l'impératrice Zita, quatre ans et demi après l'ébauche de sa réforme constitutionnelle pour François-Ferdinand et seulement quelques semaines avant la chute de l'Empire, en employant un vocabulaire napoléonien ("Illyrie»), des idées vagues, en se trompant sur la Hongrie (où auront lieu, deux semaines plus tard, puis en mars 1919, les premières révolutions d'inspiration bolchevique dans les territoires habsbourgeois) : finalement, il utilisera un autre projet, produit par les radicaux allemands et Seidler, et ne fera appel à Eichhoff que pour le style. Eichhoff lui apportera ce document après ces changements purement cosmétiques et ne le verra plus jamais ${ }^{31}$.

Force est de constater que, la situation étant déjà perdue, le projet de réforme de Charles est malgré tout moins radical et courageux que bien des ébauches ordonnées et considérées par son oncle FrançoisFerdinand avant la guerre. Des notes personnelles de Charles sur la reconstruction constitutionnelle de son empire datant de décembre 1914 - alors qu'il croyait encore à la victoire des puissances centrales et entendait même s'attribuer des colonies britanniques et françaises n'étaient guère originales, inspirantes ou prometteuses ${ }^{32}$. Il est d'autant plus consternant de noter que les promesses du manifeste d'octobre 1918 (alors que Charles n'a plus rien à perdre) ne répondent même pas aux demandes exprimées par les parlementaires non germaniques lors de la réouverture du Reichsrat en mai 1917, près de dix-huit mois auparavant. La réaction de la presse est partout glaciale, à titre d'exemple on lit dans l'éditorial de la très loyale Neue Freie Presse:

30. "Machen Sie mir wieder eine neue Textierung des Manifestes - Deutschösterreich, Böhmen, Illyrien, Halicz - wer sich dann dagegen auflehnt, gegen den wird vorgegangen, die Bolschewikenwirtschaft muss aufhören. - aber lassen Sie mir Ungarn in Ruhe. Ungarn ist der ruhende Punkt. »

31. Johann von Eichhoff, Mémoires, op. cit., p. 46-48; Bernard Michel, op. cit., p. 216.

32. Elisabeth Kovács (éd.), Untergang oder Rettung der Donaumonarchie? Politische Dokumente zu Kaiser und König Karl I. (IV.) aus internationalen Archiven, Wien, Böhlau, vol. 2, 2004, doct. 3, 13 octobre 1914-24 décembre 1914, p. 49-86. 
Pourquoi le président du conseil, malgré l'échec des consultations avec les présidents des groupes parlementaires, s'est-il décidé à une telle politique ? Il se peut que ce manifeste soit aussi destiné au président Wilson. L'État fédéral, les États-Unis d'Autriche, le nom même est un hommage à Wilson. Ainsi nous avons un ministère Wilson, nommé Hussarek ${ }^{33}$.

Pour le Fremdenblatt, cette réorganisation sans la Hongrie ne peut s'apparenter qu'à du " pur rafistolage ». La Neue Zeitung va droit au but en déclarant qu'avec la seule fédéralisation de l'Autriche, la monarchie des Habsbourg ne sera pas servie et n'en tirera pas parti. Les journaux non germanophones y prêtent à peine attention. Le Právo lidu pragois, social-démocrate, plus concerné par le succès de la grève générale du 14 octobre, déclare : "Seuls quelques jours nous séparent du moment où nous prendrons en main la gestion de nos propres affaires. » Slovenec à Ljubljana/Laibach érige les Tchèques en exemple et considère que la république a été proclamée en Bohême. Dans la même ville, le Slovenski Narod déplore qu'avec les Slaves du Sud, le gouvernement soit allé d'erreur en erreur ${ }^{34}$. Les Polonais, eux, sont agacés et sans illusions : le Kurjer Lwowski (de Lwów/Lviv/Lemberg) commente :

L'idée de restructurer l'Autriche contre les principes de Wilson [les Polonais se voient privés de la Galicie orientale] n'aura qu'un résultat : le retard d'un accord de paix. Toutes les nations d'Autriche (sauf les Allemands) s'y opposent. [...] Ce sont les méthodes utilisées par Metternich - il semble que les hommes d'État autrichiens n'aient rien appris ${ }^{35}$.

La presse hongroise, elle, est divisée entre les conservateurs au pouvoir (selon lesquels dès que la fédéralisation de l'Autriche adviendra, seule une union personnelle entre Vienne et Budapest pourra exister) et les réformateurs, qui considèrent que le parlement de Budapest n'est représentatif ni du peuple, ni du front, ni de l'arrière-pays, et demandent des droits constitutionnels égaux pour les minorités ${ }^{36}$.

33. "Warum hat der Ministerpräsident trotz des Mißerfolges in den Besprechungen mit den Obmännern zu einer solchen Politik geraten? Es mag sein, daß dieses Manifest auch an den Präsidenten Wilson gerichtet ist. Der Bundesstaat, die Vereinigten Staaten von Österreich, schon der Name ist eine Verbeugung vor Wilson. So haben wir ein Ministerium Wilson, genannt Hussarek» (NFPM, 18 octobre 1918, p. 1).

34. "Ein vollständiges Flickwerk», Auszug aus der Inlands-Presse, K. u. K. Kriegspessequartier, Wien, 18 octobre 1918, p. 2-4.

35. Kurjer Lwowski, Wydanie Wieczorne (éd. du soir), Lwów/Lviv/Lemberg, 18 octobre 1918, p. 1.

36. Auszug aus der Inlands-Presse, 18 octobre 1918, p. 4. 
Naturellement, ce document de la onzième heure est déjà largement débordé par les avancées des mouvements nationaux indépendantistes et par les projets des Alliés, qui ne sont pas dupes. Wilson va encore plus loin : les quatorze points sont caducs et seule l'autodétermination des peuples (sélective toutefois, excluant tacitement les perdants, les minorités des pays Alliés et les colonies) le satisfera. Les Autrichiens sont choqués et catastrophés, et ils accusent les Tchécoslovaques et les Yougoslaves, peuples fraîchement créés ${ }^{37}$.

Effectivement, deux jours après la signature du manifeste, les expatriés tchèques Tomáš Masaryk (dont le fils a combattu pour l'Empire) et Edvard Beneš, ainsi que leur collègue slovaque Milan Štefánik, avaient obtenu le soutien de la France pour former le gouvernement en exil de la Tchécoslovaquie, un concept sans fondement historique, inconnu et inenvisageable en 1914, et même début 1917. Ses citoyens ne sont plus des sujets habsbourgeois ${ }^{38}$. Quelques jours après la publication du manifeste, Charles avouera apparemment à Václav Klofáč - qui fut incarcéré sans procès en 1914 pour haute trahison puis amnistié par ses soins après trois ans - que l'alliance avec l'Allemagne fut fatale au pays, de même que les persécutions endurées par la nation tchèque, selon lui la plus grosse stupidité politique. Klofáč (dont le fils vient de mourir sur le champ de bataille) ne manqua pas de pointer du doigt les actions délétères des gouvernements précédents et surtout de l'ancien ministre des Affaires étrangères Ottokar von Czernin ; Charles se contenta de dire que les circonstances étaient bien trop compliquées (sans doute pour lui, en effet). Tous deux s'accordèrent à souhaiter que le sang ne coule pas $^{39}$. De leur côté, les germanophones ne tardèrent pas à suivre l'exemple de leurs ennemis jurés. Le 21 octobre, dans l'auditoire de la Diète de Basse-Autriche, 210 de leurs députés (sur les 516 parlementaires que compte le Reichsrat, le Parlement impérial) formèrent une Assemblée nationale provisoire de l'Autriche allemande (Deutschösterreich) hétéroclite et sans majorité, avec les chrétienssociaux traditionnellement monarchistes, les sociaux-démocrates et les pangermanistes, afin de défendre leurs intérêts domestiques et extérieurs. Avec la création de ce nouvel État, l'Empire est de facto liquidé, alors que le Parlement impérial à Vienne, à quelques pas de là,

37. Neue Freie Presse, Nachmittagblatt (NFPN), 21 octobre 1918, p. 1-2. Le message officiel est publié le 18 octobre par le Département d'État des États-Unis.

38. Elisabeth Kovács (éd.), op. cit., vol. 2, doct. 115, 18 octobre 1918, p. 400-401.

39. Arthur Graf Polzer-Hoditz, Kaiser Karl, op. cit., p. 558-559. 
siège encore ${ }^{40}$. Le même jour que la formation de l'Autriche allemande, Charles préside un Conseil de la couronne, durant lequel l'impasse complète dans laquelle se trouve l'Empire est dûment constatée ${ }^{41}$. Comme l'écrit le correspondant de la Neue Freie Presse:

Les Allemands d'Autriche furent toujours ceux qui s'acquittèrent des obligations d'État pour les autres. Ils en ont assez d'être les gardiens d'une maison abandonnée de tous. Les adieux sont douloureux, mais nécessaires. Le rêve de la vieille Autriche est terminé ${ }^{42}$.

Alors que l'Autriche impériale n'existe plus et que leur impopularité ne cesse de croître, Charles et Zita quittent Vienne pour inaugurer la nouvelle université de Debrecen en Hongrie de l'est, à $500 \mathrm{~km}$ de la capitale autrichienne ${ }^{43}$. Certes, au Parlement de Budapest le comte István Tisza (chef de la majorité parlementaire, homme de fer de la politique hongroise et pilier du dualisme, limogé par Charles en avril 1917) a déclaré que le Compromis de 1867 était désormais périmé, et le Premier ministre hongrois Wekerle a annoncé que le manifeste signifiait la séparation effective de l'Autriche et de la Hongrie (ne laissant subsister qu'une union personnelle) ${ }^{44}$; mais l'empereur et l'impératrice participent de plein cour aux festivités provinciales, aussi grandioses qu'incongrues. Lors du banquet, vingthuit discours se succèdent et tous proclament leur loyauté, dévotion, amour, abnégation et vénération envers le couple royal. Mais tout ceci n'est qu'une chimère. Dans le même temps, le 23 octobre à Budapest, la révolution est en marche, le gouvernement de Wekerle remet sa demande de démission. Le jour suivant, celle-ci est acceptée

40. NFPM, 22 octobre 1918, p. $2-5$; 23 octobre, p. 3 ; Stenographische Protokolle über die Sitzungen des Abgeordnetenhauses des Reichsrates, sessions 92-95, p. 4629-4701. Le Reichsrat siégera véritablement encore deux fois : le 22 (même si les Tchèques et les Slaves du Sud quittent la salle) et le 25 octobre. Le 30 octobre et 12 novembre ne seront que des réunions fantômes.

41. NFPM, 22 octobre 1918, p. 6 ; Miklós Komjáthy, Protokolle, op. cit., doct. 40, p. 690 ; doct. 41, p. 696-703 ; Arthur Freiherr Arz von Straußenburg, op. cit., p. 327.

42. "Die Deutschen in Österreich waren immer diejenigen, die zugunsten der anderen die Staatspflichten erfüllten. Sie haben es satt, die Wächter eines Hauses zu sein, das von allen verlassen ist. Der Abschied ist schmerzlich, aber notwendig. Der Traum des alten Österreich ist ausgeträumt » (NFPM, 22 octobre 1918, p. 2).

43. Haus-, Hof- und Staatsarchiv (HHStA), Nachlass Wilhelm Möller (NMö), Karton I, Akt 1-5, p. 554.

44. NFPM, 17 octobre 1918, p. 3 ; NFPA, 17 octobre, p. $2 ; 19$ octobre, p. 1. 
par Charles, et Burián, ministre commun des Affaires étrangères, abandonne à son tour son poste ${ }^{45}$. En quittant Debrecen, le prince Ludwig Windischgraetz - un proche hongrois de l'empereur tente d'expliquer à Charles l'acuité de la situation. L'empereur-roi, toujours aussi étrangement décontracté, lui rétorque : "Pourquoi [êtes-vous] si sombre ? Tout ira bien. » Lorsque son interlocuteur lui dit brutalement que tout est fini, Charles lui répond avec désinvolture : «Vous êtes un pessimiste. Tout le monde le dit. » Un échange brusque s'ensuit : Windischgraetz prévient Charles : "Majesté, vous jouez avec votre trône. Nous sommes devant l'abîme... ", et lui explique les mesures susceptibles de sauver l'Empire, notamment une paix séparée immédiate. Quand Charles tente timidement de lui répondre que "dans les prochains jours... ", Windischgraetz l'interrompt abruptement : «Non, pas dans les prochains jours, pas demain : votre Majesté doit prendre une décision ici et maintenant ${ }^{46}$. " Charles, épuisé et indécis, n'agit pas. Paradoxalement, alors que Zita semble entrevoir une lueur d'espoir dans l'allégresse qui se manifeste en ville, Charles la prévient : "Non, les acclamations ne peuvent rien arrêter maintenant ${ }^{47}$. »Charles maintient souvent l'art de l'illusion et de la minimisation devant ses collaborateurs, ses soutiens et ses sujets (et se convainc parfois lui-même), mais jamais devant sa femme.

Le couple se rend ensuite au château de Gödöllö, en périphérie de Budapest. Il s'agit de former un nouveau gouvernement, mais surtout de renforcer les liens avec la Hongrie afin d'y garder le trône royal, car Charles maintient malgré tout un certain optimisme pour cette moitié du pays, alors que tout est, pour le moment semble-t-il, perdu en Autriche ${ }^{48}$. Ce projet n'est théoriquement pas inconcevable : la

45. NFPM, 24 octobre 1918, p. 1-3; NFPA, 24 octobre, p. 1-2.

46. Stefan Kray, op. cit., p. 170-171; Ludwig Windischgraetz, Vom Roten zum Schwarzen Prinzen: Mein Kampf gegen das k. u. k. System, Berlin/Wien, Ullstein \& Co., 1920, p. 335338. Les mémoires de Windischgraetz, accrocheurs et passionnants, sont toutefois à prendre avec prudence - ils furent d'ailleurs reniés par les Habsbourg. En effet, il se donne souvent le beau rôle et s'accorde sans doute trop d'importance. Léchange fut le suivant : «Karl: „Warum so finster? Es wird alles gut werden. [...] Sie sind ein Pessimist. Alle sagen es“. Windischgraetz: „Majestät, Sie spielen mit ihrem Thron. Wir stehen vor einem Abgrund!“ Karl: „Ich werde in den nächsten Tagen.... Windischgraetz: „Nein, nicht in den nächsten Tagen, nicht morgen; Majestät müssen sich jetzt, hier, entscheiden." "

47. Gordon Brook-Shepherd, op. cit., p. 188.

48. Josef Schneider (éd. et trad.), Kaiser Franz Joseph I. und sein Hof. Erinnerungen und Schilderungen aus den nachgelassenen Papieren eines persönlichen Ratgebers, Wien/ Hamburg, Paul Zsolnay, 1984, p. 271 (réimpression). 
Hongrie restera une monarchie (sans monarque toutefois) jusqu'en 1946 et Charles y tentera deux restaurations au cours de l'année 1921. Mais concrètement, fin octobre 1918 à Gödöllö, le roi n’y fait que de la représentation et reste politiquement inefficace. Dans une atmosphère presque frivole, trois jours durant, il reçoit sans cesse. $\mathrm{Du}$ Premier ministre hongrois démissionnaire au candidat à ce poste vacant soutenu par le peuple, jusqu'à d'illustres inconnus, en passant par des membres du gouvernement, Charles reçoit 16 hommes politiques en audience pour la seule journée du 26 - en vain ${ }^{49}$. Charles déclare à Zita : "Il est toujours difficile de trouver un équipage pour un navire qui coule ${ }^{50}$. Ce n'est pas entièrement vrai : en effet, malgré la pression populaire, il ne veut, car il le considère trop instable et trop radical, accepter la nomination de l'aristocrate devenu socialdémocrate, le pacifiste comte Mihály Károlyi, qui demande le retour des troupes hongroises et la rupture de l'alliance avec Berlin. Qui plus est, le 24 octobre, les Italiens ont lancé une dernière offensive contre l'armée impériale et royale ${ }^{51}$.

Depuis Vienne, la drôle d'aventure de Charles en Transleithanie a des airs de fuite à Varennes et fait encore plonger sa déjà piètre popularité. Même en Hongrie, Charles n'est pas en sécurité. Budapest est en ébullition. On dit Gödöllő menacée ${ }^{52}$. Charles et Zita profitent d'un retour au calme temporaire pour finalement rentrer à Vienne dans la nuit du 26 au 27, laissant malgré le danger leurs enfants à Gödöllö, afin de démontrer leur confiance en la loyauté du peuple hongrois par peur des événements en Autriche (même si ce départ fait répandre des rumeurs en Transleithanie selon lesquelles Charles a fui la Hongrie $\left.^{53}\right)$. Par précaution, ils voyagent avec Károlyi, un

49. NMö, p. 556 ; Gordon Brook-Shepherd, op. cit., p. 188 ; Stefan Kray, op. cit., p. 175 ; NFPM, 25 octobre 1918, p. 1 ; 27 octobre, p. 4 ; Pester Lloyd, Morgenblatt (PLM), Budapest, 27 octobre, p. 5.

50. Gordon Brook-Shepherd, op. cit., p. 188.

51. Edmund Glaise-Horstenau (éd.), Österreich-Ungarns letzter Krieg 1914-1918, Wien, Verlag der Militärwissenschaftlichen Mitteilungen, 1930-1939, 7 vol., vol. 7 : Das Kriegsjahr 1918, 1938, p. 598 ; NMö, p. 553.

52. Josef Schneider (éd. et trad.), Kaiser Franz Joseph I., op. cit., p. 271. Selon la source anonyme du livre de Schneider (de fiabilité variable), Charles avait, durant son séjour, mené des négociations secrètes avec les Slaves du Sud et les Tchèques, leur promettant des portions de territoire hongrois afin de prévenir leur sécession - ce double jeu sébruita, et précipita apparemment son départ.

53. Ibid.; Gordon Brook-Shepherd, op. cit., p. 188-189 et 191 ; Stefan Kray, op. cit., p. 176-177. Les enfants seront malgré tout « rapatriés » à Schönbrunn quatre jours plus tard, le 31. 
bouclier humain, pour ainsi dire. S'étant résigné à nommer ce dernier, Charles fait marche arrière à la dernière seconde, sous la pression du nouveau ministre des Affaires étrangères, le compte Gyula Andrássy le jeune (pourtant beau-père de Károlyi). À sa place, et à la surprise générale, il tournera le dos aux "radicaux » et choisira János Hadik le 29 , un membre sans distinction de la noblesse terrienne, dont le mandat ne durera que deux jours ${ }^{54}$. Entre-temps, il avait nommé le bien aimé et très respecté archiduc Joseph-Auguste en tant qu'homo regius de Hongrie avec pour but de surmonter la crise politique avec les divers partis parlementaires, soutenir le nouveau gouvernement et s'adresser au peuple ${ }^{55}$. Il était bien trop tard pour ces belles intentions. La révolution (dite " des asters ») éclata le 28 quand une meute fondit sur le palais de l'archiduc pour demander la nomination de Károlyi ; la garde tira, faisant huit morts et soixante blessés. Lorsque Charles finit par nommer Károlyi - qui avait été accueilli en héros par la foule à son retour de Vienne - à la place de Hadik le 31 octobre, il est trop tard ; la révolution est certes finie (pour l'instant), mais le sang a coulé (y compris celui de Tisza, assassiné dans sa villa par des soldats révolutionnaires le $31^{56}$ ). Dans la foulée, la Hongrie déclare son indépendance. L'Autriche-Hongrie est dissoute.

De retour à Vienne, Charles avait là aussi tenté plusieurs manœuvres pour sauver son trône ${ }^{57}$. Le 27 octobre, Charles avait pris soin d' informer son «cher ami » l'empereur d'Allemagne Guillaume II de la fin de leur alliance et du commencement des négociations pour une paix séparée, car (après presque deux ans) «l'inutile effusion de sang serait un crime que [sa] conscience [lui] interdit de commettre». Il pense aussi à sa propre position quand il ajoute que : «L'ordre intérieur et le principe monarchique sont en sérieux danger ${ }^{58}$. » Puis, il envoie dans la nuit une

54. Gordon Brook-Shepherd, op. cit., p. 188 ; NFPM, 27 octobre 1918, p. 4 ; NFPN, 28 octobre, p. 2 ; NFPA, 31 octobre, p. 1.

55. NFPN, 28 octobre 1918, p. 2 ; NFPM, 29 octobre, p. 6 ; NFPA, 29 octobre, p. 2 ; PLM, 28 octobre, p. 2.

56. PLM, 28 octobre, p. 2 ; Gordon Brook-Shepherd, op. cit., p. 189-190 ; Stefan Kray, op. cit., p. 178. Zita, elle, maintint qu'aucune balle n'avait était tirée pour sauver la monarchie, contrairement aux ordres de Charles.

57. Josef Schneider (éd. et trad.), Kaiser Franz Joseph I., op. cit., p. 271. Toujours selon la source anonyme éditée par Schneider, Charles aurait apparemment eu recours aux mêmes tactiques qu'en Hongrie avec les Slaves du Sud et les Tchèques (alors qu'un gouvernement républicain provisoire est déjà en place pour l'Autriche germanophone).

58. « [...] da unnützes Blutvergießen ein Verbrechen wäre, das zu begehen Mir Mein Gewissen verbietet. Die Ordnung im Innern und das monarchische Prinzip sind in der ernstesten 
offre de paix à Wilson dans laquelle toutes les demandes américaines sont acceptées et la séparation avec Berlin annoncée ${ }^{59}$. La réaction fait l'effet d'une bombe dans les cercles militaires austro-hongrois, et nombre d'Autrichiens germanophones y voient une odieuse trahison $^{60}$. Peine perdue : l'Autriche allemande est en marche depuis une semaine lorsque la Tchécoslovaquie voit le jour le 28 octobre ; puis, le lendemain, les députés polonais annoncent le rattachement de leurs territoires (Galicie et Lodomérie) à une Pologne qui va réapparaître sur la carte après 123 ans d'absence. Simultanément, les Slaves du Sud de la Monarchie s'unissent, amputant ainsi non seulement l'Autriche et la Hongrie, mais intégrant aussi toute la Bosnie-Herzégovine. Les Italiens, quant à eux, considèrent que leurs territoires ne font déjà plus partie de la monarchie. Par ailleurs, le gouverneur de la Bucovine cède bientôt la région aux représentants des nations roumaine et ukrainienne. La Neue Freie Presse note avec sarcasme : "En Autriche, la séparation des pouvoirs, mais pas dans le sens de Montesquieu, bat son plein ${ }^{61}$. " L'Autriche impériale meurt et le 27, Hussarek laisse comme prévu sa place de Premier ministre à Heinrich Lammasch, qui n'a pour mission que de liquider l'Empire dans le calme ${ }^{62}$. Mais Charles, qui n'a ni voulu ni pu combattre et a ainsi évité une effusion de sang, n'est pas entièrement en mesure d'y croire. Trois jours plus tard, pourtant, l'Assemblée nationale provisoire annonce la création de la République d'Autriche allemande (qui comprend les territoires germanophones réclamés par la Tchécoslovaquie, les Sudètes). La dynastie n'est même pas mentionnée. Karl Renner devient chancelier. Début novembre, le nouveau Conseil d'État et l'ancien gouvernement impérial assurent une passation de pouvoir sans heurts ${ }^{63}$.

Charles ne sait plus à quel saint se vouer. Politiquement, il ne donne plus les ordres, il les reçoit. Le $1^{\text {er }}$ novembre, Joseph l'appelle au téléphone depuis Budapest et le presse d'abdiquer sa couronne de roi de Hongrie. Consternation, la conversation s'interrompt. Andrássy, présent auprès du couple royal, appelle le ministre de l'Intérieur

Gefahr... », Elisabeth Kovács (éd.), op. cit., vol. 2, doct. 118, 27 octobre 1918, p. 403-404.

59. Ibid., doct. 119, 28 octobre 1918, p. 404.

60. NMö, p. 554.

61. "In Österreich ist die Teilung der Gewalten, aber nicht die im Sinne von Montesquieu, in vollem Zuge. » NFPM, 27 octobre 1918, p. 4 ; NFPM, 29 octobre, p. 1-3 et 5-6; NFPA, 29 octobre, p. 1 ; Kurjer Lwowski, Wydanie Poranne (édition du matin), 30 octobre, p. 1.

62. NFPM, 26 octobre 1918, p. 1-2; NFPN, 28 octobre, p. 2.

63. NFPM, 31 octobre 1918, p. 1. 
hongrois, le comte Tivadar Batthyány, qui le somme de faire renoncer Charles à sa couronne, sans quoi, il sera chassé « comme un mauvais domestique ». L'archiduc Joseph reprend le combiné, annonce que des manifestations ont éclaté devant chez lui et devant le palais royal et que, faute d'abdication, le sang coulera encore. Charles se ressaisit et déclare fermement : "Le roi couronné de Hongrie n'abdiquera pas ! " Le chef de l'état-major, Arthur Arz von Straußenburg, prend la parole et annonce sombrement à Charles : "Votre Majesté, il n'y a rien à faire, tout est fini ${ }^{64}$. »

Le 28 octobre, Charles avait déjà ordonné que son pays - du moins ce qui en restait - entame des négociations avec l'Italie en vue d'un armistice $^{65}$. Le 2 novembre dans l'après-midi, les représentants du nouveau Conseil d'État se présentent à Schönbrunn et sont invités à discuter des modalités de l'armistice avec l'Italie dans le salon chinois bleu du palais. Personne ne veut en prendre la responsabilité, surtout que les conditions italiennes sont draconiennes. Charles annonce ses propres conditions et explique la situation de l'armée. Silence. Viktor Adler, le chef mourant du parti socialiste, l'interrompt : « Nous n'avons pas déclaré cette guerre. Ceux qui doivent prendre la responsabilité de cet armistice doivent être ceux qui sont responsables de cette guerre. » Charles rétorque : «Moi non plus je n’ai pas déclaré la guerre. Malgré tout, la paix doit être redonnée au peuple. » Adler accepte l'argument de l'empereur, mais lui rappelle que François-Joseph a déclaré la guerre sans consulter le peuple ou ses représentants. Il ajoute que Charles a poursuivi la guerre sans mot dire après la mort de son grandoncle. Il en conclut que les hommes présents ne sont pas en mesure de " prendre la responsabilité d'une telle monstruosité alors que le rideau s'abat ${ }^{66} "$. Charles insiste sur la gravité de la situation militaire : le front se désintègre, il est attaquable de partout, les troupes hongroises rentrent au pays - un armistice doit être conclu immédiatement. Mais Charles entonne ensuite un refrain bien familier : lui-même ne peut signer cet armistice compte tenu de sa « relation » avec Guillaume II. Qui, d'ailleurs, voudrait signer ? Les membres du Conseil d'État prennent finalement congé de l'empereur. Ce dernier convoque alors un Conseil de la couronne qui accepte l'idée d'un armistice. Charles informe alors l'état-major avant de se retirer dans le boudoir de

64. Ludwig Windischgraetz, op. cit., p. 412-414.

65. Arthur Freiherr Arz von Straußenburg, op. cit., p. 345.

66. Ibid., p. 362-366 ; NMö, p. 573-574 ; Gordon Brook-Shepherd, op. cit., p. 195-197. 
Zita. Celle-ci est furieuse et lui ordonne « de ne pas se résigner sans résistance au malheur qui s'abat ». Mais, après une discussion animée, Charles prévaut sur sa femme ${ }^{67}$.

Malgré cela, un des épisodes les plus navrants du règne de Charles s'ensuivit. Ayant ordonné tôt dans la nuit du 3 novembre que toutes les conditions de l'armistice soient acceptées et que l'armée dépose les armes immédiatement, Charles fut pris de panique, car son ordre n'avait pas le soutien du gouvernement et n'avait reçu aucune réponse des Italiens. Une demi-heure après son premier message, Charles donna donc un contrordre ; mais l'armistice avait débuté du côté austro-hongrois. Afin de ne pas être tenu responsable de l'imminente débâcle et ne pas avoir à signer le document officiel, il se délia de son rôle de généralissime de l'armée impériale et royale (dont il s'était fièrement emparé dès le 2 décembre 1916). Il céda sa position au chef de l'état-major général, Arz qui, manquant tout autant de courage, légua concomitamment son rôle de chef de l'état-major général au maréchal Hermann Kövess von Kövessháza, engagé dans une cause perdue dans les Balkans et inconscient de ces machinations ${ }^{68}$. Qui plus est, l'état-major de l'armée ne sait pas que l'armistice ne prendra effet que vingt-quatre heures après sa signature. Les Autrichiens déposent donc les armes lorsque l'armistice est signé à la Villa Giusti près de Padoue, mais les Italiens profitent des vingt-quatre heures supplémentaires pour faire 425000 prisonniers austro-hongrois ${ }^{69}$. Le 6 novembre, Charles ordonne la démobilisation de l'armée, qui sombre dans le chaos, le pillage, la violence, la peur, la colère et le désespoir. Les officiers fuient, se cachent ou se déguisent, craignant la fureur de la soldatesque ${ }^{70}$.

S'étant débarrassé de la désastreuse affaire de l'armistice (ayant effectivement déshérité et abandonné son armée, sans l'avoir remerciée ${ }^{71}$ ), Charles songe maintenant à la sécurité de sa famille, car Schönbrunn est en danger ${ }^{72}$. Alors que tous autour de lui semblent

67. NMö, p. 574-575.

68. NMö, p. 576 ; Arthur Freiherr Arz von Straußenburg, op. cit., p. 366-368.

69. Arthur Freiherr Arz von Straußenburg, op. cit., p. 370-371; Max Schiavon, L'AutricheHongrie dans la première guerre mondiale. La fin d'un empire, Saint-Cloud, Soteca 14-18, 2011, p. 240-246.

70. NMö, p. 576 ; Arthur Freiherr Arz von Straußenburg, op. cit., p. 369.

71. Alan Sked, The Decline and Fall of the Habsburg Empire, 1815-1918 [1989], London, Routledge, 2001, p. 274.

72. Stefan Kray, op. cit., p. 172. 
perdre la tête, Charles maintient son calme. Windischgraetz pénètre dans le château et est surpris de le trouver quasiment seul ${ }^{73}$. L'empereur, qui a tant hésité et si peu agi politiquement, se démène désormais pour sauver les biens de la famille. Deux vitrines du trésor impérial sont promptement retirées de la Hofburg et mises à l'abri en Suisse ${ }^{74}$. Malgré les rumeurs, la révolution attendue ne se produit pas et la famille impériale reste. D'après Zita, la présence continue de l'empereur au palais signifiait un minimum d'ordre et d'orientation ${ }^{75}$. D'ailleurs, la famille impériale refuse toujours de penser à l'impensable.

Le 8 novembre, Lammasch et son gouvernement se rendent à Schönbrunn pour donner leur démission à l'empereur ; celui-ci refuse, car si le gouvernement impérial disparaît, Charles sait qu'il suivra ${ }^{76}$. Redlich, le ministre des Finances, résume l'atmosphère dans son journal intime :

J'avais le sentiment de participer à une scène de tragédie historique, mais pas grandiose. Les ravissantes peintures rococo sur les murs, les magnifiques tapis et plafonds dans les salles de ce palais, construit par Charles VI et MarieThérèse, occupé en vainqueur par Napoléon, héberge maintenant le pauvre jeune empereur, dont le trône a probablement déjà été renversé par cette terrible guerre ! [...] l'empereur ne possède plus la moindre trace de la puissance de la dynastie, pas plus à Vienne qu'à Prague, Budapest ou Zagreb ${ }^{77} \ldots$

Tandis que les Wittelsbach abdiquent à Munich et les Hohenzollern à Berlin (les 8 et 9 novembre respectivement), le nouveau gouvernement del'Autriche allemande prend les rênes del'administration. Lammasch et son ministre de l'intérieur Edmund von Gayer se rendent de nouveau à Schönbrunn pour expliquer à Charles que l'écroulement des

73. Ludwig Windischgraetz, op. cit., p. 403 ; Gordon Brook-Shepherd, op. cit., p. 204-206.

74. Pour l'histoire du trésor impérial, voir Alphonse de Sondheimer, Vitrine XIII: Geschichte und Schicksal der österreichischen Kronjuwelen, Wien/Hamburg, Paul Zsolnay, 1966.

75. Gordon Brook-Shepherd, op. cit., p. 206.

76. Fritz Fellner et Doris Corradini (éd.), Schicksalsjahre Österreichs: Die Erinnerungen und Tagebücher Josef Redlichs 1869-1936, Wien, Böhlau, 2011, 3 vol., vol. 2, p. 465 (JR).

77. "Ich hatte das Gefühl, an einer Szene einer historischen aber nicht großartigen Tragödie teilzunehmen. Die reizenden Rokokogemälde an den Wänden, die wundervollen Tapeten, und Plafonds in den Sälen dieses Schlosses, das Karl VI, und Maria Theresia gebaut, das Napoleon als Sieger bewohnte, beherbergt jetzt den armen jungen Kaiser, dessen Thron dieser furchtbare Krieg wohl schon umgestürtzt hat! [...] da der Kaiser keine Spur mehr von der Macht des alten Erzhauses besitzt, weder in Wien noch in Prag, weder in Budapest noch in Agram... » (JR, vol. 2, p. 464). 
dynasties voisines ne sera pas sans conséquence à Vienne. L'officier, historien et archiviste Edmund Glaise-Horstenau note que, pour la première fois, pour un court moment, le calme aplomb qui dominait chez le jeune prince durant ses jours heureux, chancela; que son visage perdit de sa couleur et qu'un tremblement parcourut son corps ${ }^{78}$. Malgré cela, Charles refuse d'abdiquer. Le Conseil d'État (y compris les chrétiens-sociaux traditionnellement monarchistes) veut pourtant une république.

Le 10 novembre, alors qu'il ne lui reste qu'un jour à régner, Charles est un homme vaincu. Lors de sa prière du matin à Schönbrunn, il est " pâle, grisonnant et visiblement ému ${ }^{79}$ ». À midi, Lammasch et Gayer sont de nouveau présents, cette fois pour présenter à Charles l'ébauche d'un manifeste selon lequel Charles (sans toutefois abdiquer) renonce à participer aux affaires d'État et accepte de laisser le peuple décider de la forme de gouvernement de son choix. Charles veut tout d'abord y réfléchir sans pression politique et fait temporairement sortir les deux ministres. Son secrétaire personnel, Karl von Werkmann, reste à ses côtés et le presse de signer. Mais, comme souvent, Charles ne veut ni ne peut décider seul, et fait appeler Zita. Celle-ci s'y oppose avec véhémence. L'impératrice survole le texte et affronte son mari, indignée et furieuse :

Jamais - tu ne peux pas abdiquer... Un empereur ne peut jamais abdiquer. Il peut être déposé, il peut être déchu de ses droits de souverain. D'accord. C'est la méthode violente. Mais celle-ci ne le force pas à reconnaître la perte de ses droits. Il peut l'attaquer en justice, au gré du moment et des circonstances, mais abdiquer : jamais, jamais, jamais. Je préfère périr ici avec toi - Otto [leur fils aîné] viendra ensuite. Et si nous devons tous périr ensemble - il y a d'autres Habsbourg ${ }^{80}$ !

78. "Zum ersten Mal kam die ruhige Fassung, die den in glücklichen Tagen knabenhaft jugendlichen Fürsten beherrschte und auch von den Gegnern ohne Vorbehalt anerkannt wurde, für einen kurzen Augenblick ins Wanken. Sein Antlitz verfäbte sich, ein Zittern lief über seinen Körper » (Edmund Glaise-Horstenau, Die Katastrophe, Die Zertrümmerung Österreich-Ungarns und das Werden der Nachfolgestaaten, Wien, Amalthea, 1929, p. 441).

79. «bleich, ergraut, sichtlich ergriffen » (ibid., p. 445).

80. "Niemals kann ein Herrscher abdanken. Er kann abgesetzt, kann seiner Herrscherrechte verlustig erklärt werden. Gut. Das ist Gewalt. Sie verpflichtet ihn nicht zur Anerkennung, daß er seine Rechte verloren habe. Er kann sie verfolgen, je nach Zeit und Umständenaber abdanken - nie, nie, nie! Lieber falle ich mit dir hier, dann wird Otto kommen. Und wenn wir alle fallen sollten - noch gibt es andere Habsburger! » (Karl Werkmann, Der Tote auf Madeira, München, Kulturpolitik, 1923, p. 17-19). 
Cette sortie ne sert à rien : les deux ministres attendent avec impatience une réponse. Finalement, Gayer ouvre la porte lui-même. Charles et Zita se retirent alors avec Werkmann dans la salle de porcelaine du palais pour parvenir à une décision. Werkmann fait valoir à Charles l'argument selon lequel une Autriche impériale obtiendrait nécessairement une mauvaise paix lors des négociations. Charles, qui semble avoir perdu de sa lucidité, l'interrompt :

Fadaises ! Je suis sûr que je peux apporter plus à l'Autriche, qu'on veut m'entendre moi, plutôt que ces messieurs qui ont poussé la folie jusqu'à irriter les vainqueurs avec leur déclaration en faveur d'un Anschluss. Cette Autriche sera terrassée à Paris ${ }^{81}$.

Werkmann contre avec conviction. Charles demande timidement : " N'y a-t-il pas même une petite force qui serait prête à défendre la monarchie? "Werkmann répond par la négative. Charles comprend finalement et acquiesce. Dans le salon chinois bleu du palais, Werkmann annonce à Lammasch et Gayer que la proclamation peut être publiée ${ }^{82}$. La déclaration est brève, mais non dénuée de force :

Depuis mon accession au trône, je n'ai cessé de m'efforcer d'extraire mes peuples des horreurs de la guerre, pour laquelle je ne porte aucune responsabilité. [...] Comme toujours rempli d'un amour immuable pour tous mes peuples, je ne veux pas poser ma personne en obstacle à leur libre épanouissement. [...] Je renonce à toute participation aux affaires de l'État. [...] Seule la paix intérieure peut guérir les blessures de cette guerre ${ }^{83}$.

Mais Charles est têtu et n'accepte finalement pas la valeur légale ou définitive de ses mots. Dans son esprit, il est toujours empereur de

81. «Das ist ein Unsinn! Sicher ist, daß ich Österreich mehr bringen kann, daß man auf mich eher hören wird als auf die Herren, die den Irrsinn so weit treiben, die Sieger durch eine Anschlußerklärung zu reizen. Dieses Österreich wird in Paris zu Boden getreten werden » (ibid., p. 19-20).

82. "Gibt es keine noch so kleine Macht, die willens wäre, die Monarchie zu verteidigen? " (ibid., p. 20-22).

83. "Seit Meiner Thronbesteigung war Ich unablässig bemüht, Meine Völker aus den Schrecknissen des Krieges herauszuführen, an dessen Ausbruch Ich keinerlei Schuld trage. [...] Nach wie vor von unwandelbarer Liebe für alle Meine Völker erfüllt, will Ich ihrer freien Entfaltung Meine Person nicht als Hindernis entgegenstellen. [...] Ich verzichte auf jeden Anteil an den Staatsgeschäften. [...] Nur der innere Friede kann die Wunden dieses Krieges heilen» (NFPM, 11 novembre 1918, p. 1). 
droit divin. Le 12 novembre, il déclare à Ludwig von Flotow, le dernier ministre des Affaires étrangères de la double monarchie, qu'il n'a signé ce manifeste que sous la contrainte et qu'il ne pouvait donc accepter la situation juridique qui en résultait. Charles, toujours dans le déni, rédige d'ailleurs un papier dans lequel il désavoue sa proclamation précédente. Flotow refuse de le contresigner et conseille à Charles de le détruire. Charles, comme à son habitude, va consulter Zita. Lorsqu'il revient après quelques minutes, il a encore une fois changé d'avis. Il suit la recommandation de Flotow et brûle ce morceau de papier dans la cheminée ${ }^{84}$.

À 15 heures, les ministres arrivent à Schönbrunn avec la version finale du manifeste afin que Charles la signe officiellement. Ce dernier le fait ostensiblement au crayon de papier, un geste peut-être symbolique (même s'il signait habituellement de cette manière). Il signera une déclaration similaire pour les Hongrois deux jours plus tard. En début de soirée, après une dernière prière avec les enfants dans la chapelle du palais, tous les membres de la famille impériale se réunissent dans le salon des cérémonies pour faire leurs adieux à leurs fidèles serviteurs ${ }^{85}$. Sept voitures attendent la famille en habits civils pour l'emmener en sûreté, au château d'Eckartsau, à environ $50 \mathrm{~km}$ à l'est de la capitale. Le jour suivant, le 12 novembre, la République d'Autriche est proclamée à l'unanimité par le groupement national provisoire. En mars 1919, avec tous les biens de la dynastie étant sur le point d'être confisqués ${ }^{86}$ et la sécurité de ses membres impossible à garantir, Charles et sa famille quitteront le pays.

Même si cet article se limite aux dernières semaines du règne de Charles, il révèle de nombreuses facettes typiques du personnage, souvent les moins glorieuses. Mais avant de porter un jugement final sur cet homme à la fin tragique (fauché par la maladie en exil à Madère en 1922, à 34 ans, laissant sept enfants et une impératrice enceinte), il convient de reconnaître sa modestie, son charme personnel, son dévouement, sa fidélité, son intelligence au-dessus de la moyenne, ses moments de

84. Erwin Matsch (éd.), November 1918 auf dem Ballhausplatz. Erinnerungen von Ludwig Freiherr von Flotow, des letzten Chefs des österr.-ungarischen Auswärtigen Dienstes 18951920, Graz, Böhlau, 1982, p. 332.

85. Stefan Kray, op. cit., p. 178-179 et 181.

86. Staatsgesetzblatt für den Staat Deutschösterreich, 71. Stück (10 avril 1919), n 209 : "Gesetz vom 3. April 1919, betreffend die Landesverweisung und die Übernahme des Vermögens des Hauses Habsburg-Lothringen », p. 513-514. 
lucidité, son dégoût face aux effusions de sang malgré son courage et son talent de soldat, et surtout son humanité. Certaines de ces qualités furent sans doute fatales pendant son règne ; certaines relevaient purement de l'innocence, de la faiblesse et surtout de la naïveté. Comme le fait remarquer Jean Sévillia, Charles manifeste " une étonnante confiance dans le genre humain ${ }^{87}$ ». Qui plus est, il faut admettre que pendant son dernier mois sur le trône, nul ne pouvait sauver l'Autriche-Hongrie ou le trône des Habsburg depuis Vienne. À l'extérieur (les Alliés) comme à l'intérieur (les nationalités), on souhaitait la liquidation de l'Empire. Les dés étaient jetés depuis un certain temps.

Contrairement à ce que véhiculent certaines idées reçues, Charles était un personnage assez ambigu : à la fois impétueux et indécis, insouciant et réfléchi, léger sans être frivole, conscient, mais peu consciencieux. À sa décharge, il fut pendant tout son règne coincé dans une situation inextricable. Il a souvent été dit de lui, y compris par ses détracteurs, qu'il aurait fait un empereur honnête et compétent en période de paix. Mais cette spéculation n'a pas lieu d'être : Charles ne connut que la guerre. Certes, il savait dès 1900, après le mariage morganatique de François-Ferdinand, qu'il serait un jour empereur, mais personne (y compris lui-même) ne semble avoir réfléchi en profondeur à ce sujet. Avant Sarajevo, Charles pensait avoir encore vingt ou trente ans pour se préparer ${ }^{88}$. Même à la fin juin 1914, alors que seul un vieil homme fatigué et fataliste le séparait du trône et que la guerre était imminente, nul n'intervint pour le former à son rôle futur, voire très proche. Charles, se considérant avant tout comme un soldat, voulait combattre, et il n'apprit ni ne maîtrisa l'art de gouverner. Il lisait peu et ne s'intéressait pas aux détails. La mort de son grand-oncle le prit de court - pour beaucoup, François-Joseph était devenu un personnage immortel. Ainsi, en novembre 1916, à 29 ans, fraîchement devenu empereur et roi, Charles paraissait être le premier surpris. Tout ceci lui fut fatal, car sa seule marge de manœuvre politique, si elle existait, se présenta dans les premiers mois de son règne (même si son couronnement en Hongrie avait rapidement limité son pouvoir de réforme à la Cisleithanie) $)^{89}$.

Il ne considéra la réforme constitutionnelle de son Empire qu'en temps de crise, pris de panique : en avril et mai 1917, après la première

87. Jean Sévillia, Le dernier empereur, op. cit., p. 204.

88. Gordon Brook-Shepherd, op. cit., p. 3.

89. Ceci est l'argument central de la thèse de doctorat de l'auteur, déjà citée. 
révolution russe et la déclaration de guerre américaine à l'Allemagne, puis en octobre 1918. Même à ces moments, il ne songeait qu'à son trône et son Empire, et donc à la paix (au-delà de ses préoccupations humanitaires, qui jouèrent certes un rôle, mais moindre). Entre ces crises, il adopta la politique autrichienne classique du fortwursteln (qui consistait à avancer lentement, en se débrouillant tant bien que mal). Pour un homme qui, en tant qu'héritier (et plus tard en tant qu'exilé), avait reconnu les dangers fatals du dualisme, de la germanisation et de la magyarisation dans un pays démographiquement de plus en plus slave, il est étonnant de constater qu'après quelques semaines au pouvoir il nomma un gouvernement pro-allemand en Autriche et accéda aux demandes magyares en se faisant couronner à Budapest. Dès juin 1917, il avait reconvoqué le Parlement viennois et limogé ses deux Premiers ministres, mais il était déjà trop tard et leurs remplaçants n'avaient aucune envergure (alors que les candidats de qualité, sans être légion, existaient). Sa faiblesse l'empêcha aussi d'imposer le projet progressiste et plausible de son ami et chef de cabinet Polzer-Hoditz; il finira même par le renvoyer en novembre 1917, sous la pression des nationalistes allemands ${ }^{90}$. Sans doute Charles n'a-t-il jamais été le fédéraliste émancipateur et libéral de ses "mythographes ». Un an après la réouverture du Parlement, désabusé par des jeux politiques qu'il n'a jamais compris, frustré par l'absence de coopération entre les nationalités, aigri par la perte de bienveillance à son égard et fermement maintenu sous le joug de Berlin après les révélations sur ses tentatives de paix par le truchement de Sixte, il abandonna ses velléités réformistes et relança un programme germanophile. Qui plus est, Charles ne renia jamais le serment de son couronnement hongrois, et ne se désolidarisa du Reich allemand que fin octobre 1918, dans un geste futile. Jamais il n'avait sérieusement envisagé une séparation avec l'Allemagne auparavant, même s'il en avait peut-être rêvé. Aurait-il fait ces deux choses dès le début de son règne, au moins aurait-il pu explorer la possibilité de se sauver, même si une rébellion (voire une sécession) hongroise et austro-allemande, civile ou militaire, était possible ; une invasion ordonnée par Berlin, par contre, semble bien moins plausible. Il n'y a d'ailleurs aucune preuve de cette dernière, contrairement aux propos d'après-guerre de Charles, de Zita et de Czernin.

90. Christopher Brennan, Reforming Austria-Hungary, op. cit., successivement p. 303-304 et 252-255. 
Pendant ces dernières semaines, Charles est à bout de forces. Il voyage trop, il reçoit trop, il travaille trop (souvent sur des sujets sans importance) et a trop de responsabilités familiales. Malgré les ordres répétés de son médecin, il ne dort que deux ou trois heures par nuit. Il est tellement épuisé qu'il grelotte dans son bureau terriblement surchauffép $^{1}$. Il est aussi désespéré que perplexe. Il ne peut comprendre que ses sujets se tournent contre lui, sa dynastie, sa mission, comme il le confie de façon très agitée à Windischgraetz :

Moi aussi je voulais un coup d'État, et une séparation de l'Allemagne [...] Qui me soutint à l'époque? Ne voulais-je pas satisfaire les Tchèques et les Slaves du Sud? Je ne suis pas un magicien. Il est impossible que la révolution puisse se tourner contre moi. Le fait que je n'aie pas commencé plus tôt avec les reformes les plus radicales est bien la preuve que je ne suis pas un autocrate. Je suis pieds et poings liés. La politique des peuples doit être faite par les peuples ${ }^{92}$.

Cette croyance religieuse, quasi mystique dans le caractère divin, permanent et invariable de sa position, expliquent peut-être ses moments de calme pendant la tempête. Charles, et qui plus est Zita, ne crurent jamais véritablement qu'ils avaient perdu le soutien du peuple tout entier et de Dieu, surtout après la guerre, lorsqu'ils s'alimentèrent de théories du complot. Sans doute cette certitude contribua-t-elle aussi à l'étrange passivité et à l'irrésolution de Charles dans l'urgence, ce qui ne manqua pas de faciliter la décomposition puis l'oubli de l'Empire.

Deux écoles de pensée s'affrontent sur le rôle de Charles. L'une, le plus souvent monarchiste, traditionaliste et catholique - la canonisation de Charles ne saurait tarder - voient en lui un martyr qui aura lutté jusqu'au bout pour ses convictions, son pays et ses sujets. Kray, un fonctionnaire de la chancellerie, écrit : «Il ne peut honnêtement pas être dit de l'empereur Charles que, dans ces derniers jours critiques de la Monarchie, il n'a pas essayé de tout son possible d'éviter la

91. Gordon Brook-Shepherd, op. cit., p. 188 ; Karl Werkmann, Der Tote auf Madeira, op. cit., p. 14.

92. "Auch ich wollte einen Umsturz, auch ich eine Loslösung von Deutschland [...] Wer unterstützte mich damals? Wollte ich nicht die Tschechen, die Südslawen befriedigen? Zaubern kann ich nicht. Es ist unmöglich, daß die Revolution sich gegen mich kehren kann. Daß ich nicht früher mit den radikalsten Reformen kam, beweist ja eben, daß ich nicht autokratisch regieren will. Ich bin gebunden. Die Politik der Völker müssen die Völker machen " (Ludwig Windischgraetz, op. cit., p. 339). 
catastrophe ${ }^{93}$. " Deux commentaires s'imposent : premièrement, Charles n'a pas tenté tout ce qui était possible et deuxièmement, s'il a effectivement tenté tout son possible, alors son aptitude à gouverner ne peut être que sérieusement mise en doute. Le second courant consiste à balayer Charles d'un revers de main. L'historien austro-américain Robert A. Kann ne lui consacra que quelques phrases de son magnum opus sur l'Empire : " [...] il échoua partout et à tous les égards", ajoutant que "presque chacune de ses tentatives désastreuses d'enrayer le désastre imminent se termina par une situation pire que si aucune tentative n'avait été faite ${ }^{94} »$. Comme le démontrent ses dernières semaines sur le trône, Charles ne correspondait ni à l'une, ni à l'autre de ces définitions.

93. Stefan Kray, op. cit., p. 175.

94. Robert A. Kann, The Multinational Empire. Nationalism and National Reform in the Habsburg Empire, New York, Columbia University Press, 1950, 2 vol., vol. 2 : Empire Reform, p. 239. 